\title{
Effect of CT misalignment on attenuation - corrected myocardial perfusion SPECT
}

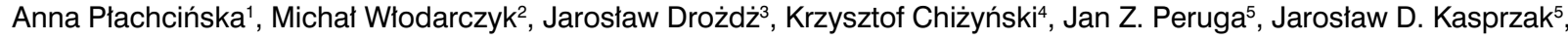 \\ Jacek Kuśmierek² \\ ${ }^{1}$ Department of Quality Control and Radiological Protection, Inter-Faculty Chair of Medical Imaging and Radiology, \\ Medical University of Łódź, Poland \\ 2Department of Nuclear Medicine, Inter-Faculty Chair of Medical Imaging and Radiology, Medical University of Łódź, Poland \\ ${ }^{3}$ Department of Cardiology, Chair of Cardiology and Cardiac Surgery, Medical University of Łódź, Poland \\ ${ }^{4}$ Department of Intensive Cardiac Therapy, Chair of Cardiology and Cardiac Surgery, Medical University of Łódź, Poland \\ ${ }^{5}$ Department of Cardiology, Chair of Cardiology, Medical University of Łódź, Poland
}

[Received 5 V 2015; Accepted 26 VI 2015]

\begin{abstract}
BACKGROUND: Use of CT based attenuation correction (AC) for myocardial perfusion SPECT (MPS) is growing fast due to a rapid development of hybrid SPECT/CT systems. SPECT and CT studies are performed in a sequential way extending total study acquisition and making a patient movement more likely. The present work aims at answering the question how large misalignment between SPECT and CT studies should be considered significant and how often those misregistrations are observed. MATERIAL AND METHODS: A retrospective study applying AC was performed in 107 patients who had coronary angiography (CA) performed within 3 months. Patients underwent a stress/rest Tc-99m MIBI 2 day SPECT/CT myocardial perfusion study. In case of SPECT and CT misalignment CT slices were shifted manually; shifts along 3 axes were recorded and after realignment a repeat reconstruction was performed. Euclidean distance of misalignment was also calculated. Images were analyzed by two experienced nuclear medicine specialists (consensus) applying visual semiquantitative method. Perfusion of three arteries was scored using a 5 grade scale. CA results were used as a reference for MPS findings.

RESULTS: In 47 patients (44\%) CT realignment was necessary. CT was shifted mostly along $x$ and y axes, and less often along $z$ axis. Euclidean distance $S$ exceeded 2 pixels in 3 stress and 2 rest studies. Only in 7 patients changes of scores assigned to coronary vessels were noted as a result of CT realignment. These changes concerned 9 vessel areas. In 7 out of 9 cases changes were noted toward a better agreement with results of CA. Only in one patient, with stress $S>3$ pixels and negative result of CA, CT realignment changed vessel area score significantly, from probably abnormal to normal.

CONCLUSIONS: Only misalignments large enough, exceeding 2-3 pixels, have negative impact on attenuation corrected images. Such misalignments are rare, in our material were observed in 3 stress and 2 rest studies (3\% and $2 \%$ of all studies, respectively). Only in one patient (below $1 \%$ of all studied patients) CT misalignment caused a significant study misinterpretation. Although alignment of SPECT and CT studies should be checked in every patient, small misalignments do not affect study interpretation.
\end{abstract}

KEY words: attenuation correction, myocardial perfusion study, SPECT and CT misalignment

Nuclear Med Rev 2015; 18, 2: 78-83

\section{Background}

Myocardial perfusion SPECT (MPS) is a widely accepted diagnostic tool applied in the management of patients with coronary artery disease. This imaging modality plays an important role in diagnosing cardiovascular disease, establishing prognosis, assessing the effectiveness of therapy, and evaluating viability. Whereas MPS is a valuable diagnostic tool, it is also a complex

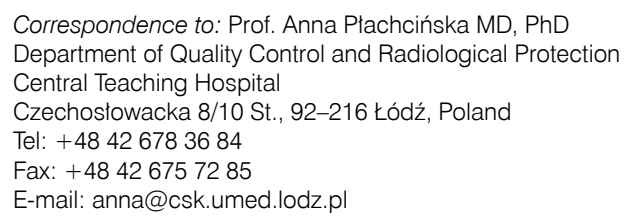

imaging process, which exposes it to several potential pitfalls and artifacts that can limit the utility of the study [1]. One important source of artifacts is attenuation of gamma rays inside a patient body. For this reason attenuation correction is one of key factors affecting diagnostic accuracy of the method. At first several types of systems with transmission hardware and external radionuclide sources were applied for this purpose [2-6]. Recently, as a result of a rapid development of hybrid SPECT/CT systems, attenuation correction of myocardial perfusion studies makes use of maps obtained by transmission of X-rays [7-10]. This CT-based correction has a major advantage over radionuclide source methods: a much higher number of collected photons resulting in better quality of correction maps and therefore also attenuation — corrected SPECT images. On the other hand, SPECT and CT studies are performed in a sequential way extending total study acquisition and making 
a patient movement more likely. In the literature several publications on frequency and severity of misalignment between SPECT and CT studies as well as major sources of artifacts in corrected images can be found [11-18]. Some of those studies made use of heart phantoms [16], another ones of patient studies [13-15, 17] or both - phantoms and patients [11, 12, 18]. Authors of those studies present very diverse opinions on the effect of misalignment between SPECT and CT studies on attenuation - corrected perfusion images. Some of them $[11,12]$ claim that even a misalignment as small as half a pixel can cause visible differences between attenuation - corrected images before and after CT and SPECT alignment. On the other hand, Kennedy et al. [13] present opinion that only misalignments as large as 3 pixels should be considered significant. To our knowledge, no communication verifying those opinions concerning SPECT and CT misalignment is available yet.

The present work aims at answering the question how large misalignment should be considered significant. The study uses myocardial perfusion images of patients with known results of coronary angiography, without post-infarction scars, in order to avoid ambiguity caused by additional perfusion defects in images.

\section{Material and methods}

A retrospective analysis of 107 patients with known or suspected $\mathrm{CAD}$, without clinical history, electrocardiographic or echocardiographic signs of a previous myocardial infarction or any other factors affecting myocardial perfusion, like cardiomyopathy, severe aortic valve disease or left bundle branch block, referred for stress-rest MPS in the years 2010-2013 was performed. All patients underwent coronary angiography less than 3 months before or after MPS. A study group consisted of 65 males and 42 females. The clinical and demographic characteristics of patients are presented in Table 1. The study was approved by the Medical University Bioethics Committee.

\section{Study protocol}

Patients underwent a stress/rest 2 day myocardial perfusion imaging. In 82 (77\%) patients an exercise stress test according to Bruce protocol was performed and the remaining ones had a dipyridamole infusion (0.56-0.84 mg/kg in $4 \mathrm{~min}$.). Beta blockers, nitrates, calcium channel blockers and trimetazidine were discontinued 48 hours before a stress study. Radiopharmaceutical — technetium-99m methoxy-isobutyl-isonitryl (MIBI), in activity of $0.3 \mathrm{mCi}(11 \mathrm{MBq})$ per kilogram of body mass, was administered intravenously at peak stress or 3-6 minutes after dipyridamole infusion. After administration of a radiopharmaceutical patients ate a fatty meal to accelerate hepatobiliary clearance of the tracer. Patients were instructed not to move during the whole study.

\section{Table 1. Characteristics of patients}

\begin{tabular}{lc} 
Parameter & Number \\
\hline Number of patients & 107 \\
Males & 65 \\
Females & 42 \\
Mean age (years) & $62 \pm 8$ \\
Mean BMl $\left[\mathrm{kg} / \mathrm{m}^{2}\right]$ & $28.5 \pm 4.5$ \\
Mean echocardiographic ejection fraction [\%] & $61 \pm 7$
\end{tabular}

Stress study acquisition was started 45 minutes and rest study - 1 hour after administration of a radiopharmaceutical. Studies were performed with a hybrid Infinia Hawkeye (GE) camera, equipped with high resolution collimators. Study protocol consisted of acquisition of 60 projections in a matrix $64 \times 64$ (zoom 1.28, pixel size $6.9 \mathrm{~mm}$ ). After completion of emission study a low dose CT study of thorax was conducted $(140 \mathrm{KV}, 2.5 \mathrm{~mA})$ in order to obtain a map of attenuation coefficients. SPECT studies were reconstructed with OSEM reconstruction method (2 iterations, 10 subsets), applying attenuation correction. Every time an OSEM reconstruction with attenuation correction was applied, an alignment of emission and transmission slices was visually checked. In case of misalignment CT slices were manually shifted making use of an ACQC tool; shifts along three axes ( $z, y, x)$ were recorded (in millimeters) and after a proper alignment a repeat reconstruction was performed. In addition, the Euclidean distance of misalignment in all 3 dimensions of space (square root of a sum of squares of shifts along 3 axes) was calculated. A technician reconstructing studies was unaware of results of patients' coronary angiography.

\section{Image analysis}

Reconstructed slices were analyzed by two nuclear medicine specialists (a consensus) applying a visual semiquantitative method. The method was based on a standard assignment of myocardium to three main coronary arteries, in a way recommended by the American Heart Association [19]. Additionally, all slices in two perpendicular planes (sagittal and coronal) were also inspected according to the areas of coronary blood supply. Perfusion of three arteries was scored with the use of a 0 to 4 numerical scale: 0 - definitely normal, 1 - probably normal, 2 - equivocal, 3 - probably abnormal and 4 - definitely abnormal. When a CT study needed alignment with SPECT study, attenuation corrected images obtained before and after CT realignment were displayed next to each other to allow for a direct comparison between them. Interpreters were unaware of results of coronary angiography.

\section{Coronary angiography}

Invasive contrast coronary angiography was performed according to standard percutaneous techniques, with each arterial segment visualized in at least 2 perpendicular planes. Angiograms were analyzed by experienced angiographers unaware of SPECT imaging findings. Significant CAD was defined as $\geq 70 \%$ luminar diameter narrowing by visual inspection in at least one of the three coronary arteries and $\geq 50 \%$ in the main left coronary artery. Coronary angiography findings were subsequently used as a reference for the results of attenuation-corrected myocardial perfusion study.

\section{Statistical analysis}

Data were described in terms of frequencies of occurrence and also mean values and standard deviations.

\section{Results}

\section{Coronary angiography}

Coronary angiography revealed no critical stenosis in 65 patients and in the remaining 42 - critical stenosis of 1 (28 patients), 2 (11, including 2 patients with critically stenosed left main artery) and 3 (3 patients) coronary arteries. 


\section{Myocardial perfusion imaging}

In 47 patients (44\% of the whole studied group) CT realignment was considered necessary. CT shift was carried out mostly along $x$ and $y$ axes (Table 2), and less often along $z$ axis. CT realignments were made along axes in both directions, although in case of $x$ axis (left-right) mostly in positive (left) direction (52 from among 58 all shifts along $x$ axis), along y axis also mostly in positive (dorsal) direction (41 of 50 all shifts along this axis), whereas in case of shifts along $z$ axis - in majority of cases the shift was carried out in negative (cephalad) direction (in 24 of 27 cases). Mean and maximal CT shifts in positive direction of $x$ axis (left) were equal to $5,2 \mathrm{~mm}$ ( 0.75 pixel) and 16,2 mm (2.4 pixels), respectively, in positive (dorsal) direction of y axis $-5.3 \mathrm{~mm}(0.8$ pixel) and $8.2 \mathrm{~mm}$ (1.2 pixel), respectively, and in negative (cephalad) direction of $\mathrm{z}$ axis $-7,5 \mathrm{~mm}$ (1.1 pixel) and $23 \mathrm{~mm}$ (3.3 pixels), respectively. Realignment of CT study was necessary in 39 stress and 41 rest studies; in 33 patients realignment of both studies was necessary, in 6 - of only stress and 8 - only rest. Realignment of CT was made most frequently along 2 axes (in 53\% of all studies; Table 2), less frequently along one (29\%) and least often along all 3 (19\% of studies) axes. Mean and maximal Euclidean distance of CT shift in stress studies were equal to $8.53 \mathrm{~mm}$ (1.24 pixel) and $45.6 \mathrm{~mm}$ (6.6 pixels), respectively and in rest studies $-6.3 \mathrm{~mm}$ (0.92 pixel) and $21 \mathrm{~mm}$ (3.1 pixels), respectively. CT vs. SPECT realignment Euclidean distances in stress and rest studies are presented in Table 3. CT realignment was necessary in 15 patients with positive and 32 patients with negative result of coronary angiography.

From among 47 patients in whom CT realignment was considered necessary only in 7 patients changes of scores assigned to coronary vessel areas were noted (Table 4). These changes concerned 9 vessel areas. In 2 patients (Table 4, no. 1 and 2) without

Table 2. Directions and numbers of axes along which CT studies were realigned

\begin{tabular}{|lccc|}
\hline $\begin{array}{l}\text { Directions (axes) } \\
\text { of realignment }\end{array}$ & $\begin{array}{c}\text { Number of } \\
\text { stress studies }\end{array}$ & $\begin{array}{c}\text { Number of } \\
\text { rest studies }\end{array}$ & Together \\
\hline$x$ & $29(74 \%)^{\star}$ & $29(71 \%)$ & $58(73 \%)$ \\
$y$ & $27(69 \%)$ & $23(56 \%)$ & $50(63 \%)$ \\
\hline$z$ & $10(26 \%)$ & $17(41 \%)$ & $27(34 \%)$ \\
\hline Number of axes & & & \\
\hline 1 & $10(25 \%)$ & $13(32 \%)$ & $23(29 \%)$ \\
\hline 2 & $23(59 \%)$ & $19(46 \%)$ & $42(53 \%)$ \\
3 & $6(15 \%)$ & $9(22 \%)$ & $15(19 \%)$ \\
\hline
\end{tabular}

*Per cent values calculated in relation to numbers of studies with necessary CT realignment (39 stress and 41 rest studies), $x$ — left-right, y - ventro-dorsal, z — caudal-cephalad directions stenoses of coronary arteries CT realignment modified slightly scores assigned to LAD blood supply area, from probably to definitely normal. In the third patient (Table 4, no. 3) a change of score assigned to $\mathrm{Cx}$ area was significant, from probably abnormal to definitely normal. In case of a patient no. 4 , with a $50 \%$ stenosis of the left main coronary artery and $90 \%$ ostial stenosis of $L A D$, score assigned to left anterior descending artery remained the same - abnormal after CT realignment and was minimally changed in Cx area. In a patient no. 5 with positive result of coronary angiography (occluded RCA) CT realignment changed the RCA score from equivocal to probably abnormal. In a patient no. 6 with a $70 \%$ stenosis of a dominant $\mathrm{Cx}$, right after branching of a first marginal artery being also stenosed ostially in $75 \%$, CT realignment changed slightly an area score (from 4 to 3 ) which was assigned in both cases to RCA (differentiation between RCA and Cx areas in myocardial perfusion study is difficult in some patients). In the last patient (no. 7) with $75 \%$ stenosis of RCA, CT realignment did not change a score (abnormal) assigned to this vessel area, but enhanced doubts concerning LAD area (shift of a score from rather abnormal to equivocal) and reduced doubts (from equivocal to rather normal) concerning $\mathrm{Cx}$ artery area. In effect, out of 9 changes of scores assigned to vessel areas as a result of CT realignment, in 7 cases changes were noted toward a better agreement with results of coronary angiography (meaning the best agreement to be 0 for unstenosed and 4 for stenosed vessel).

Only in one patient (no. 3), with negative result of coronary angiography, CT realignment changed a score assigned to a vessel (CX) significantly, from 3 (probably abnormal) to 0 (normal). Euclidean distance of CT misalignment in this patient was equal to 3.3 pixels in stress study and 1.9 pixels in rest study (Fig. 1). On the other hand, in a patient no. 6, with the largest CT misalignment (stress and rest Euclidean distances equal to 6.6 and 3.2 pixels, respectively) it affected only slightly scores assigned to particular arteries. This effect was observed in a patient with a positive result of coronary angiography, with evident perfusion defects in uncorrected study, which were only slightly affected by attenuation correction. In the only patient with normal coronary vessels and stress CT misalignment equal to 2 pixels (Table 3 ) and rest -1.7 pixels no visible difference before and after CT realignment in attenuation corrected images was observed.

In summary, in the studied material of 107 patients changes of scores assigned to coronary artery areas as a result of CT realignment were observed in 7 (6.5\%) patients. Although CT misalignment exceeded 1 pixel in stress study of 21 patients and rest study of 18 patients (Table 3 ), only in one patient (below $1 \%$ of all studied patients) CT misalignment caused a significant study misinterpretation. This patient formed $1.5 \%$ of all patients with negative

Table 3. Misalignment of CT vs. SPECT studies

\begin{tabular}{lccccc} 
& \multicolumn{3}{c}{ Numbers of misaligned studies } \\
\cline { 2 - 3 } & \multicolumn{2}{c}{ All patients } & & \multicolumn{2}{c}{ Patients without critical stenoses of coronary vessels } \\
\cline { 2 - 3 } & Stress study & Rest study & & Stress study & Rest study \\
$\mathrm{S}<1$ pixel & $18(46 \%)^{*}$ & $23(56 \%)$ & $11(44 \%)$ & $18(58 \%)$ \\
1 pixel $\leq \mathrm{S}<2$ pixels & $18(46 \%)$ & $16(39 \%)$ & $12(48 \%)$ & $12(39 \%)$ \\
2 pixels $\leq \mathrm{S}<3$ pixels & $1(3 \%)$ & $1(2 \%)$ & $1(4 \%)$ & $1(3 \%)$ \\
$\mathrm{S} \geq 3$ pixels & $2(5 \%)$ & $1(2 \%)$ & $1(4 \%)$ & $0(0 \%)$
\end{tabular}

*Per cent values calculated in relation to numbers of studies with necessary CT realignment, $\mathrm{S}$ - Euclidean distance in pixels 
Table.4. List of patients in whom CT realignment changed scores assigned to three main coronary artery areas. Directions of realignment: $+x$ left, $-x$ right; +y dorsal, $-y$ ventral; +z caudal, $-z$ cephalad. Only in one patient (no. 3) CT realignment significantly changed a Cx score, from 3 (probably abnormal) to 0 (normal)

\begin{tabular}{|c|c|c|c|c|c|c|c|c|c|c|c|c|c|c|c|c|c|}
\hline \multirow[b]{4}{*}{ 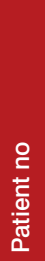 } & \multicolumn{14}{|c|}{ Myocardial perfusion study } & \multicolumn{3}{|c|}{ Coronary angiography } \\
\hline & \multirow{2}{*}{\multicolumn{3}{|c|}{$\begin{array}{c}\text { CT } \\
\text { before realignment }\end{array}$}} & \multirow{2}{*}{\multicolumn{3}{|c|}{$\begin{array}{c}\text { CT } \\
\text { after realignment }\end{array}$}} & \multicolumn{8}{|c|}{ CT realignment } & \multirow[b]{3}{*}{$\frac{9}{4}$} & \multirow[b]{3}{*}{ త } & \multirow[b]{3}{*}{ ঠ্ৰ } \\
\hline & & & & & & & \multicolumn{4}{|c|}{ Stress } & \multicolumn{4}{|c|}{ Rest } & & & \\
\hline & 号 & త & ర্ৰ & 号 & ơ & ర্ৰ & $\begin{array}{l}\frac{\text { क }}{d} \\
\frac{x}{a} \\
\frac{x}{x}\end{array}$ & $\begin{array}{l}\frac{\Phi}{\Phi} \\
\text { 즐 } \\
\text { 을 }\end{array}$ & 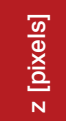 & 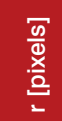 & 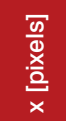 & $\begin{array}{l}\frac{\bar{g}}{9} \\
\frac{x}{2} \\
\text { 은 }\end{array}$ & 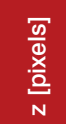 & $\begin{array}{l}\frac{\bar{d}}{d 0} \\
\text { 흘 } \\
=\end{array}$ & & & \\
\hline 1. & 1 & 0 & 0 & 0 & 0 & 0 & 0 & 0 & 0 & 0.0 & 0 & 0 & 0.4 & 0.4 & - & - & - \\
\hline 2. & 1 & 0 & 0 & 0 & 0 & 0 & 0.8 & -0.6 & 0.5 & 1.2 & 0.7 & -0.2 & 0.7 & 1 & - & - & - \\
\hline 3. & 0 & 3 & 0 & 0 & 0 & 0 & 2.4 & 0 & -2.3 & 3.3 & 0.8 & 0 & -1.7 & 1.9 & - & - & - \\
\hline 4. & 4 & 2 & 0 & 4 & 1 & 0 & 1 & 0 & -1 & 1.4 & 0.8 & 0 & -1 & 1.3 & + & - & - \\
\hline 5. & 0 & 0 & 2 & 0 & 0 & 3 & 0.5 & 0.5 & 0 & 0.7 & 0.6 & 0 & -0.5 & 0.8 & - & - & + \\
\hline 6. & 0 & 1 & 4 & 0 & 0 & 3 & -5.5 & 1.5 & -3.3 & 6.6 & -2 & 0.8 & -2.3 & 3.1 & - & + & - \\
\hline 7. & 1 & 2 & 4 & 2 & 1 & 4 & 0.8 & 1 & 0 & 1.3 & 0.8 & 0.8 & 0 & 1.2 & - & - & + \\
\hline
\end{tabular}

+ critically stenosed vessel, - no critical stenosis

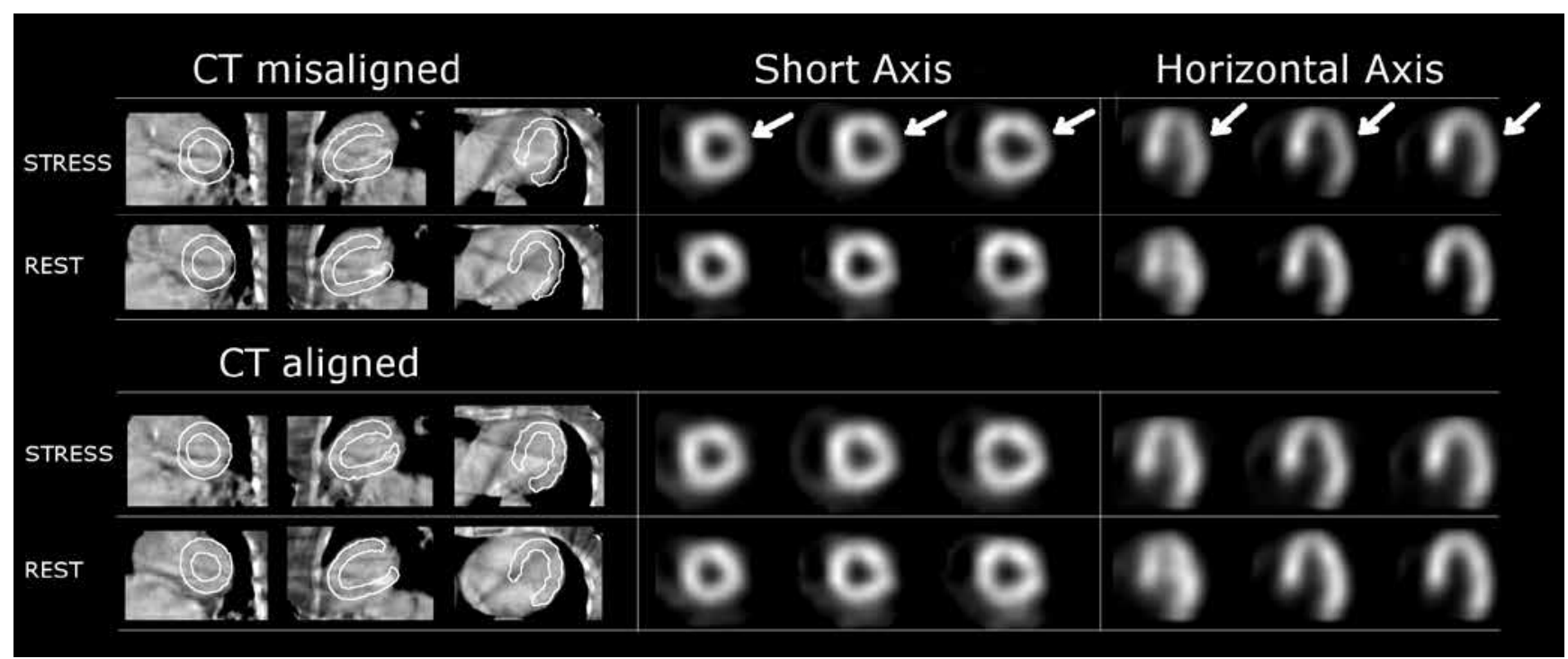

Figure 1. Effect of CT misalignment and CT realignment on myocardial perfusion images. Overlapping of myocardium in SPECT study with left lung in CT caused a false defect in attenuation corrected perfusion images (arrows). After CT realignment perfusion defect disappeared (patient no. 3 in Table 4)

results of coronary angiography and $2 \%$ of patients with necessary CT realignment.

\section{Discussion}

Although many articles validating attenuation correction applied to myocardial perfusion imaging can be found in the literature [2, 7 , 20-22], publications questioning a positive impact of this correction, at least in some subgroups of patients, can be also found [3, 5, 8, $9,23]$. In spite of these objections application of attenuation correction becomes widely practiced, in compliance with the common guidelines of the European Association of Nuclear Medicine, the European Society of Cardiac Radiology and the European Council of Nuclear Cardiology [24].
Under these circumstances it appears necessary to determine whether misalignment of CT and SPECT studies affects attenuation corrected perfusion images and how large misregistration should be considered significant. The question how large misalignment between SPECT and CT studies changes interpretation of attenuation corrected images has not been determined so far. Recent publications on the question of misalignment between imaging modalities in myocardial perfusion studies concern only PET/CT images $[25,26]$.

Unlike other surveys on the effect of CT and SPECT misalignment on attenuation corrected myocardial perfusion images, our study was performed in a group of patients with known results of coronary angiography, which were later used as a verification method for the correctness of CT realignment, that appears to be a very subjective 
action. The study was made on a material of patients without a history of myocardial infarction in order to allow unequivocal evaluation of the impact of CT and SPECT misalignment on the agreement of attenuation corrected study outcome with coronary angiography. Post-infarction scars could hinder this evaluation.

The present study provided information on frequency and magnitude of CT and SPECT misalignment. Generally, authors of communications on this issue agree that this misalignment is observed often, from $20.5 \%$ [27] through $42 \%$ ( moderate to severe misalignment) in the article by Goetze et al. [14], two third of studies with misalignment exceeding one pixel [15], to $73 \%$ of studies with misalignment larger than one pixel and $23 \%$ with misalignment exceeding two pixels [13]. Our results are within the published data, with frequency of misalignment exceeding $40 \%$. A significant dispersion of published frequencies can be attributed to different experience of technicians reconstructing studies, various populations of patients, different study protocols (1-day protocol may be more exhausting for a patient and make him more prone to movement). Significant differences between published data can be also caused by a subjectivity of a manual method of SPECT and CT realignment, with additional suboptimal quality of low-dose CT. In spite of efforts made toward automation of CT and SPECT realignment [28] automatic methods for realignment are still not offered in commercially available software used for reconstruction of myocardial perfusion SPECT.

CT images needed shifts mostly in a left side (along $x$ axis), in a dorsal side (along y axis) and in a cephalad side (along $z$ axis). Misalignment along $x$ axis was probably an effect of patient movement, along z axis - could also result from patient breathing movement. However, misalignment along y axis, in majority (about 4/5) of cases in a ventral direction, can be probably explained by additional deflection of a bed being placed in a position proper for CT study.

Realignment of CT images first of all was made in order to avoid overlapping of myocardium in SPECT study on lungs on CT images. This overlapping could distort attenuation corrected perfusion images due to a substantially lower density of this organ. In case of patient movement in a right direction (along $x$ axis) this is especially true for a lateral wall of myocardium and in a caudal direction (along z axis) — for anterior wall. In general, it is important for a myocardium in SPECT image not to overlap evidently with a left lung on CT study because it is a source of improper attenuation correction due to a lower attenuation coefficient of the lung. In our material substantial part of changes of artery scores caused by CT realignment (Table 4) was noted in antero-septal wall (supplied with blood by LAD) -3 patients and lateral wall $(C x)-4$ patients. It resulted from overlapping of myocardium in SPECT study with left lung in CT images and needed CT realignment.

In effect, although misalignment of SPECT and CT was found in a substantial part of studies, its significant impact on attenuation corrected study result was observed in only one patient $(<1 \%$ of all studied patients and $1.5 \%$ of patients without critical stenoses of main coronary arteries). In the remaining 6 patients (Table 4) the impact of CT realignment on the final outcome of attenuation-corrected SPECT study was quite insignificant. A large misalignment in one patient (Table 4, no. 6) did not change a study result significantly because this patient had a considerable perfusion defect and a positive result of a coronary angiography. In one female patient (Table 4, no. 1) a minor change of a score assigned to LAD perfu- sion area was observed although CT misalignment was found only in a rest study and it was as small as $3.6 \mathrm{~mm}$. This change might have been caused by subjective factors affecting results of a manual study processing and might not have been a direct consequence of SPECT and CT misalignment. One should keep in mind that after CT realignment a study needs to be reconstructed again, so changes in a final study outcome may also be a consequence of e.g. different orientation of myocardial axes.

However, in the remaining majority of patients with CT misalignment exceeding 1 pixel (16 out of 21 stress and 13 out of 18 rest studies, Table 3 and 4 ) no effect of CT realignment on attenuation - corrected myocardial perfusion images has been observed. It seems that our results confirm opinions presented by Kennedy et al. [13] that only misalignments exceeding 3 pixels should be considered significant rather than views shared by another authors [11, 12] that even a misalignment as small as half a pixel may be a source of significant change of tracer distribution within attenuation corrected images of a myocardium. If it were true that a misalignment smaller than one pixel significantly affected attenuation corrected images, as was shown in publications by Fricke et al. and Matsunari et al. in phantom studies [11, 12], attenuation corrected images would be affected by remarkable random errors. This point has been emphasized by Nichols in his editorial concerning this issue [29]. Results of our work show that only misalignments large enough have negative impact on attenuation corrected images. At the same time, large misalignments between CT and SPECT studies are rare; in our material misalignments exceeding 2 pixels were observed in 3 stress and 2 rest studies (3\% and $2 \%$ of all studies, respectively) and exceeding 3 pixels - in 2 stress and 1 rest studies (2\% and $1 \%$, respectively).

The fact that in 7 out of 9 vessel scores changes were noted toward a better agreement with results of coronary angiography speaks in favor of the method of CT realignment in spite of a significant subjectivity of a manual method applied for realignment of these two imaging modalities.

A method applied in our work for visual assessment of perfusion images, based on evaluation of blood supply areas of three coronary arteries has been applied in numerous articles $[2,7,8,10]$. This method is more useful in detection of small differences in tracer distribution than a segment method [19], because it takes into account all slices in three planes, not only selected transversal slices. Moreover, this way of image assessment enabled a direct comparison between myocardial perfusion images and coronary angiography which is also evaluated by arteries. Images before and after CT realignment were compared directly, side by side, otherwise small differences would have disappeared in a noise introduced by subjectivity of a visual analysis.

\section{Conclusions}

Only misalignments large enough, exceeding 2-3 pixels, have negative impact on attenuation corrected images. Such misalignments are rare, in our material were observed in 3 stress and 2 rest studies (3\% and $2 \%$ of all studies, respectively). Only in one patient ( $<1 \%$ of all studied patients) CT misalignment caused a significant study misinterpretation. Although alignment of SPECT and CT studies should be checked in every patient, small misalignments do not affect study interpretation. 


\section{References}

1. Burell S, McDonald A. Artifacts and pitfalls in myocardial perfusion imaging. J Nucl Med Technol 2006; 34: 193-211.

2. Ficaro E, Fessler J, Shreve $P$ et al. Simultaneous transmission/emission myocardial perfusion tomography: diagnostic accuracy of attenuation corrected Tc-99m sestamibi single-photon emission computed tomography. Circulation 1996; 93: 463-473. doi: 10.1161/01.CIR.93.3.463.

3. Vidal R, Buvat I, Darcourt J et al. Impact of attenuation correction by simultaneous transmission-emission tomography on visual assessment of 201TI myocardial perfusion images. J Nucl Med 1999; 40: 1301-1309.

4. Kluge R, Sattler B, Seese A, Knapp WH. Attenuation correction by simultaneous emission-transmission myocardial single-photon emission tomography using technetium-99m-labelled radiotracer: impact on diagnostic accuracy. Eur J Nucl Med 1997; 24: 1107-1114.

5. Slart RHJA, Que TH, van Veldhuisen DJ et al. Effect of attemuation correction on interpretation of 99mTc-sestamibi myocardial perfusion scintigraphy: the impact of 1 year's experience. Eur J Nucl Med Mol Imaging 2003; 30 : 1505-1509. doi 10.1007/s00259-003-1265-3.

6. Wolak A, Slomka PJ, Fish MB et al. Quantitative diagnostic performance of myocardial perfusion SPECT with attenuation correction in women. J Nucl Med 2008; 49: 915-922. doi: 10.2967/jnumed.107.049387.

7. Masood Y, Yi-Hwa L, DePuey G et al. Clinical validation of SPECT attenuation correction using $\mathrm{x}$-ray computed tomography-derived attenuation maps: Multicenter clinical trial with angiographic correlation. J Nucl Cardiol 2005; 12: 676-686. doi: 10.1016/j.nuclcard.2005.08.006.

8. Huang R, Fanglan L, Zhen Z et al. Hybrid SPECT/CT attenuation correction of stress myocardial perfusion imaging. Clin Nucl Med 2011; 36: 344-349. doi: 10.1097/RLU.0b013e318212c525.

9. Genovesi D, Giorgetti A, Gimalli A et al. Impact of attenuation correction and gated acquisition in SPECT myocardial perfusion imaging: results of the multicentre SPAG (SPECT Attenuation Correction vs Gated) study. Eur J Nucl Med Mol Imaging 2011; 38: 1890-1898. doi 10.1007/s00259-011-1855-4.

10. Sharma P, Chetan DP, Sellam K et al. Comparative accuracy of CT attenuation - corrected and non-attenuation-corrected SPECT myocardial perfusion imaging. Clin Nucl Med 2012; 37: 332-338. doi: 10.1097/RLU.0b013e31823ea16b

11. Fricke $H$, Fricke $E$, Weise $R$ et al. A method to remove artifacts in attenuation-corrected myocardial perfusion SPECT introduced by misalignment between emission scan and CT-derived attenuation maps. J Nucl Med 2004; 45: 1619-1625

12. Matsunari I, Böning G, Ziegler et al. Effects of misalignment between transmission and emission scans on attenuation-corrected cardiac SPECT. J Nucl Med 1998; 39: 411-416.

13. Kennedy JA, Israel O, Frenkel A. Directions and magnitudes of misregistration of CT attenuation-corrected myocardial perfusion studies: incidence, impact on image quality, and guidance for reregistration. J Nucl Med 2009; 50: 1471-1478. doi: 10.2967/jnumed.109.062141.

14. Goetze S, Brown TL, Lavely WC et al. Attenuation correction in myocardial perfusion SPECT/CT: effects of misregistration and value of reregistration. J Nucl Med 2007; 48: 1090-1095
15. Goetze S, Wahl R. Prevalence of misregistration between SPECT and CT for attenuation-corrected myocardial perfusion SPECT. J Nucl Cardiol 2007; 14: 200-206. doi: 10.1016/j.nuclcard.2006.12.325.

16. Mc Quaid SJ, Hutton BF. Sources of attenuation-correction artefacts in cardiac PET/CT and SPECT/CT. Eur J Nucl Med Mol Imaging 2008; 35: 1117-1123. doi: 10.1007/s00259-008-0718-0.

17. Utsunomiya D, Nakaura T, Honda T et al. Object-specific attenuation correction at SPECT/CT in thorax: optimization of respiratory protocol for image registration. Radiology 2005; 237: 662-669.

18. Takahashi Y, Murase K, Higashino H et al. Attenution correction of myocardial SPECT images with X-ray CT: effects of registration errors between X-ray CT and SPECT. Ann Nucl Med 2002: 16: 431-435.

19. Cerqueira MD, Weissman NJ, Dilsizian $V$ et al. Standardized myocardial segmentation and nomenclature for tomographic imaging of the heart: a statement for healthcare professionals from the Cardiac Imaging Committee of the Council on Clinical Cardiology of the American Heart Association. Circulation 2002; 105: 539-542. doi: 10.1161/hc0402.102975.

20. Links JM, Becker LC, Rigo $P$ et al. Combined corrections for attenuation, depth-dependent blur, and motion in cardiac SPECT: a multicenter trial. J Nucl Cardiol 2000; 7: 414-425.

21. Duvernoy CS, Ficaro EP, Karabajakian MZ et al. Improved detection of left main coronary artery disease with attenuation-corrected SPECT. J Nucl Cardiol 2000; 7: 639-648

22. Shotwell M, Singh BM, Fortman $\mathrm{C}$ et al. Improved coronary disease detection with quantitative attenuation-corrected TI-201 images. J Nucl Cardiol 2002; 9: 52-61.

23. Lee DS, So Y, Cheon GJ et al. Limited incremental values of attenuation-noncorrected gating and ungated attenuation correction to rest/stress myocardial perfusion SPECT in patients with an intermediate likelihood of coronary artery disease. J Nucl Med 2000; 41: 852-859.

24. Flotats A, Knuuti J, Gutberlet $M$ et al. Hybrid imaging: SPECT and CT. A joint position statement by the European Association of Nuclear Medicine (EANM), the European Society of Cardiac Radiology (ESCR) and the European Council of Nuclear Cardiology (ECNC). Eur J Nucl Med Mol Imaging 2001; 38: 201-212. doi: 10.1007/s00259-010-1586-y.

25. Alessio AM, Kinahan PE, Champley KM, Caldwell JH. Attenuation - emission alignment in cardiac PET/CT based on consistency conditions. Phys Med Biol 2010; 37: 1191-2000

26. Nakazato R, Dey D, Alexanderson D et al. Automatic alignment of myocardial perfusion PET and 64 slice coronary CT angiography on hybrid PET/CT. J Nucl Cardiol 2012; 19: 482-491. doi: 10.1007/s12350-012-9528-7.

27. Tonge CM, Manoharan M, Lawson RS, Shields RA, Prescott MC. Attenuation correction of myocardial SPECT studies using low resolution computed tomography images. Nucl Med Commun 2005; 26: 231-237.

28. Chen J, Caputlu-Wilson SF, Hongcheng $S$ et al. Automated quality control of emission-transmission misalignment for attenuation correction in myocardial perfusion imaging with SPECT-CT systems. J Nucl Cardiol 2006; 13: 43-49.

29. Nichols KJ. How serious a problem for myocardial perfusion assessment is moderate misregistration between SPECT and CT. J Nucl Cardiol 2007; 14: 150-152. 